Quim. Nova, Vol. 35, No. 5, 914-919, 2012

\title{
SYNTHESIS OF 2-AZETIDINONE DERIVATIVES OF 6-NITRO-1H-INDAZOLE AND THEIR BIOLOGICAL IMPORTANCE
}

\author{
Pushkal Samadhiya*, Ritu Sharma, Santosh K. Srivastava and S. D. Srivastava \\ Department of Chemistry, Dr. H.S. Gour University (A Central University), Sagar, M.P. India 470003
}

Recebido em 12/6/11; aceito em 4/12/11; publicado na web em 23/3/12

\begin{abstract}
A new series of 3-chloro-1-\{[2-(6-nitro-1H-indazol-1-yl)ethyl]amino $\}$-4-(substituted phenyl)-2-azetidinones (4a-j) was synthesized in four steps from 6-nitro- $1 H$-indazole and characterized by IR, ${ }^{1} \mathrm{H}$ NMR, ${ }^{13} \mathrm{C}$ NMR, FAB-mass spectrometry and chemical methods. Compounds $\mathbf{4}(\mathbf{a}-\mathbf{j})$ were screened in vitro for their antibacterial, antifungal and antitubercular activities against some selected microorganism and for their antiinflammatory activity (in vivo) against albino rats (either sex). All above activities of compounds 4(a-j) showed acceptable results.
\end{abstract}

Keywords: synthesis; azetidinone; biosignificance.

\section{INTRODUCTION}

Indazole nucleus represents a very attractive scaffold to obtain new molecules endowed with antiprotozoal activity. ${ }^{1}$ In the present study our research group has reported the synthesis of new indazole derivatives owing to their interesting antimicrobial, antitubercular and antiinflammatory activities. Indazoles have been found to possess promising medicinal activities such as antibacterial, antiinflammatory, inhibitors of human cytosolic phospholipase, nitric oxide synthases and antiproliferative activities. ${ }^{2-7}$

The azetidin-2-one skeleton has been recognized as a useful building block in synthesis of biologically important compounds. This is mainly the strain energy associated with the four membered azetidin-2-one ring, makes it susceptible for nucleophilic ring cleavage. This factor is also responsible for their application as synthons for various stereoselective syntheses of biological active heterocyclic compounds. $\beta$-Lactam derivatives display interesting biological activities such as CXCR2 receptor potent antagonists, antimicrobial, antitubercular and anti-HCMV activities..$^{8-13}$ As a consequence, the interest of organic chemists in the synthesis of new $\beta$-lactam derivatives remains high. ${ }^{14}$ These activities aroused our attention and prompted us to synthesize some $\beta$-lactam derivatives. In the present study, we are reporting the synthesis of some new 2-azetidinone derivatives of 6-nitro- $1 H$-indazole. All the synthesized compounds were characterized by IR, ${ }^{1} \mathrm{H}$ NMR, ${ }^{13} \mathrm{C}$ NMR, FAB-Mass and chemical methods. Compounds $\mathbf{4}(\mathbf{a}-\mathbf{j})$ have been screened in vitro for their antibacterial, antifungal and antitubercular activities against some selected microorganism and the antiinflammatory activity (in vivo) screened against albino rats (either sex). All above activities of compounds 4(a-j) showed acceptable results.

\section{RESULTS AND DISCUSSION}

3-Chloro-1- $\{[2-(6-$ nitro-1H-indazol-1-yl)ethyl]amino $\}$-4-(substitutedphenyl)-2-azetidinone, compounds $\mathbf{4}(\mathbf{a}-\mathbf{j})$ were synthesized in four different steps (Scheme 1). ${ }^{14}$ 6-Nitro- $1 H$ indazole on reaction with $\mathrm{Cl}\left(\mathrm{CH}_{2}\right)_{2} \mathrm{Br}$ at room temperature afforded 1-(2-chloroethyl)-6-nitro-1H-indazole, compound 1. IR spectrum of compound 1 displayed absorption at 1326 and $768 \mathrm{~cm}^{-1}$ for $\left(\mathrm{N}-\mathrm{CH}_{2}\right)$ and $(\mathrm{C}-\mathrm{Cl})$, respectively. This clearly indicated the

\footnotetext{
*e-mail: pushkalsamadhiya@ rediffmail.com
}

disappearance of $-\mathrm{NH}$ absorption $\left(3442 \mathrm{~cm}^{-1}\right)$. The compound 1 on reaction with hydrazine hydrate at room temperature yielded 1-(2-hydrazinoethyl)-6-nitro- $1 H$-indazole, compound 2. IR spectrum of compound 2 showed absorption peak for $\mathrm{NH}$ at $3342 \mathrm{~cm}^{-1}$ while absorption of $(\mathrm{C}-\mathrm{Cl})$ has disappeared. The ${ }^{1} \mathrm{H}$ NMR spectra of compound 2 displayed signal for $\left(\mathrm{CH}_{2}-\mathrm{NH}\right)$ at $\delta 3.30-3.34$ ppm. The compound $\mathbf{2}$ on further reaction with several substituted aromatic aldehydes produced $(E / Z)$-substituted benzaldehyde [2-(6-nitro-1H-indazol-1-yl)ethyl]hydrazones, compounds 3(a-j). For the compounds $\mathbf{3}(\mathbf{a}-\mathbf{j})$ characteristic absorption peak for Schiff base $(\mathrm{N}=\mathrm{CH})$ in IR spectra appeared in the range of $1553-1583 \mathrm{~cm}^{-1}$, the ${ }^{1} \mathrm{H}$ NMR chemical shift in the range of $\delta$ 7.89-7.98 ppm and its ${ }^{13} \mathrm{C}$ NMR signal was found in the range of $\delta 150.6-155.7 \mathrm{ppm}$. In the ${ }^{1} \mathrm{H}$ NMR a broad signal of $\mathrm{NH}_{2}$ at $\delta 5.92$ ppm disappeared. Compounds $3(\mathbf{a}-\mathbf{j})$ on treatment with $\mathrm{ClCH}_{2} \mathrm{COCl}$ in the presence of $\mathrm{Et}_{3} \mathrm{~N}$ furnished the final products, compounds $4(\mathbf{a}-\mathbf{j})$. The spectra of compounds $\mathbf{4}(\mathbf{a}-\mathbf{j})$ carbonyl group of $\beta$-lactam ring showed characteristic absorption peak in the range of $1729-1741 \mathrm{~cm}^{-1}$ and ${ }^{1} \mathrm{H}$ NMR spectra two doublet appeared for $(\mathrm{N}-\mathrm{CH})$ and $(\mathrm{CH}-\mathrm{Cl})$ in the range of $\delta$ 5.22-5.37 and 4.22-4.68 ppm respectively. In the ${ }^{13} \mathrm{C}$ NMR spectra three signals appeared for $(\mathrm{N}-\mathrm{CH}),(\mathrm{CH}-\mathrm{Cl})$ and (CO cyclic) in the range of $(\delta)$ 61.4-65.7, 50.1-53.5 and 168.1$172.7 \mathrm{ppm}$, respectively. The IR absorption and ${ }^{1} \mathrm{H}$ NMR signal for $\mathrm{N}=\mathrm{CH}$ have been disappeared. All above facts strongly indicate the synthesis of all above compounds.

\section{Pharmacological results and discussion}

A new series of 3-chloro-1-\{[2-(6-nitro-1H-indazol-1-yl)ethyl] amino -4-(substitutedphenyl)-2-azetidinone, compounds $\mathbf{4}(\mathbf{a}-\mathbf{j})$ were synthesized and screened for their antibacterial and antifungal activities against some selected bacteria and fungi respectively and antitubercular activity was screened against $M$. tuberculosis $(\mathrm{H} 37 \mathrm{Rv}$ strain). Results of antimicrobial, antitubercular and antinflammatory activities are given in Tables 1 and 2. Results of given activities revealed that the synthesized compounds $\mathbf{4}(\mathbf{a}-\mathbf{j})$ have a structure activity relationship (SAR) because the activity of them varies with substitution. Nitro group containing compounds $(\mathbf{4 h}, \mathbf{4 i}$ and $\mathbf{4 j}$ ) showed higher activity than chloro $(\mathbf{4 c}, \mathbf{4 d})$ or bromo containing compounds (4e, 4f). Chloro or bromo derivatives also have higher activity than other tested compounds.

On the basis of SAR, we concluded that the activity of compounds 
Table 1. Antibacterial, antifungal and antitubercular activities of compounds 4(a-j) with MIC value $(\mu \mathrm{g} / \mathrm{mL})$

\begin{tabular}{|c|c|c|c|c|c|c|c|c|c|}
\hline \multirow{2}{*}{ Comp. } & \multicolumn{4}{|c|}{ Antibacterial activity } & \multicolumn{4}{|c|}{ Antifungal activity } & \multirow{2}{*}{$\begin{array}{c}\begin{array}{c}\text { Antitubercular } \\
\text { activity }\end{array} \\
\text { M. tuberculosis }\end{array}$} \\
\hline & B. subtilis & E. coli & S. aureus & K. pneumoniae & A. niger & A. flavus & F. oxisporium & C. albicans & \\
\hline $4 a$ & 12.5 & $>6.25$ & 12.5 & 6.25 & $>25$ & $>25$ & $>25$ & $>25$ & $>12.5$ \\
\hline $4 b$ & $>3.25$ & 6.25 & 3.25 & $>3.25$ & $>25$ & $>12.5$ & $>25$ & $>25$ & $>2.5$ \\
\hline $4 c$ & 6.25 & $>3.25$ & 6.25 & 3.25 & $>12.5$ & 25 & $>12.5$ & $>12.5$ & $>2.5$ \\
\hline $4 d$ & $>3.25$ & 6.25 & 3.25 & 6.25 & $>12.5$ & $>25$ & $>12.5$ & $>12.5$ & 2.5 \\
\hline $4 e$ & 6.25 & $>3.25$ & 3.25 & $>3.25$ & $>12.5$ & 25 & 25 & $>25$ & $>2.5$ \\
\hline $4 f$ & 6.25 & 3.25 & 6.25 & $>3.25$ & $>12.5$ & $>12.5$ & $>12.5$ & $>12.5$ & $>2.5$ \\
\hline $4 g$ & $>3.25$ & 6.25 & $>3.25$ & 6.25 & 25 & $>12.5$ & $>12.5$ & 25 & 6.25 \\
\hline $4 h$ & 3.25 & $>3.25$ & 3.25 & 3.25 & 25 & $>12.5$ & $>12.5$ & $>25$ & 2.5 \\
\hline $4 i$ & 3.25 & 3.25 & $>3.25$ & 3.25 & $>12.5$ & $>12.5$ & $>12.5$ & $>12.5$ & 2.5 \\
\hline $4 j$ & 3.25 & $>3.25$ & 3.25 & 3.25 & $>12.5$ & $>12.5$ & $>12.5$ & $>12.5$ & $>2.5$ \\
\hline
\end{tabular}

The mic values of standard streptomycin for all bacteria strain and griseofulvin for all fungi strain were in the range of $1.25-3.25$ and $6.25-12.5 \mu \mathrm{g} / \mathrm{mL}$ respectively. Isoniazid and rifampicin were used as standards, mic values in the range of $1.25-2.50 \mu \mathrm{g} / \mathrm{mL}$ for $M$. tuberculosis.

Table 2. Antiinflammatory activity of compounds $\mathbf{4}(\mathbf{a}-\mathbf{j})$

\begin{tabular}{cccc}
\hline $\begin{array}{c}\text { Compound } \\
\text { code }\end{array}$ & $\begin{array}{c}\text { Before } \\
\text { carageenan } \\
\text { administration } \\
(\text { mean } \pm \text { SEM })\end{array}$ & $\begin{array}{c}\text { Total increase } \\
\text { in paw volume } \\
\text { after } 5 \text { h (mean } \\
\pm \text { SEM) }\end{array}$ & $\begin{array}{c}\text { Percent } \\
\text { inhibition }\end{array}$ \\
\hline $\mathbf{4 a}$ & $0.60 \pm 0.02$ & $0.16 \pm 0.02$ & 50.00 \\
$\mathbf{4 b}$ & $0.64 \pm 0.02$ & $0.14 \pm 0.02$ & 56.25 \\
$\mathbf{4 c}$ & $0.66 \pm 0.02$ & $0.13 \pm 0.01$ & 59.38 \\
$\mathbf{4 d}$ & $0.68 \pm 0.02$ & $0.13 \pm 0.02$ & 59.38 \\
$\mathbf{4 e}$ & $0.66 \pm 0.03$ & $0.14 \pm 0.02$ & 56.25 \\
$\mathbf{4 f}$ & $0.65 \pm 0.02$ & $0.12 \pm 0.01$ & 62.50 \\
$\mathbf{4 g}$ & $0.67 \pm 0.02$ & $0.13 \pm 0.01$ & 59.38 \\
$\mathbf{4 h}$ & $0.64 \pm 0.03$ & $0.12 \pm 0.01$ & 62.50 \\
$\mathbf{4 i}$ & $0.65 \pm 0.02$ & $0.10 \pm 0.03$ & 68.75 \\
$\mathbf{4 j}$ & $0.67 \pm 0.03$ & $0.11 \pm 0.02$ & 65.63 \\
Control & $0.66 \pm 0.02$ & $0.32 \pm 0.01$ & - \\
Standard; & $0.68 \pm 0.03$ & $0.08 \pm 0.02$ & 75.00 \\
\hline phenylbutazone & & & \\
\hline
\end{tabular}

depends on electron withdrawing nature of the substituent groups. The sequence of the activity is following.

$$
\mathrm{NO}_{2}>\mathrm{Cl}>\mathrm{Br}>\mathrm{H}
$$

\section{CONCLUSION}

A new series of compounds $\mathbf{4}(\mathbf{a}-\mathbf{j})$ has been synthesized successfully by a simple route and investigated for their antimicrobial, antitubercular and antiinflammatory activities. Data of all activities revealed that the compounds $(\mathbf{4 b}),(\mathbf{4 d}),(\mathbf{4 f}),(\mathbf{4 g}),(\mathbf{4 h}),(\mathbf{4 i})$ and $(\mathbf{4 j})$ displayed higher activities than the other compounds of the series.

\section{EXPERIMENTAL}

Melting points were taken in open capillaries and are uncorrected. Progress of reaction was monitored by silica gel-G coated TLC plates using $\mathrm{MeOH}: \mathrm{CHCl}_{3}$ system (1:9). The spot was visualized by exposing dry plate at iodine vapours chamber. IR spectra were recorded in $\mathrm{KBr}$ disc on a Schimadzu 8201 PC, FTIR spectrophotometer $\left(v_{\max }\right.$ in $\left.\mathrm{cm}^{-1}\right)$ and ${ }^{1} \mathrm{H}$ NMR and ${ }^{13} \mathrm{C}$ NMR spectra were measured on a Brucker DRX-300 spectrometer in $\mathrm{CDCl}_{3}$ at 300 and $75 \mathrm{MHz}$ respectively using TMS as an internal standard. All chemical shifts were reported on $\delta$ scales. The FAB-Mass spectra were recorded on a Jeol SX-102 mass spectrometer. Elemental analyses were performed on a Carlo Erba-1108 analyzer. The analytical data of all the compounds were highly satisfactory. For column chromatographic purification of the products, Merck silica Gel 60 (230-400 Mesh) was used. The reagent grade chemicals were purchased from the commercial sources and further purified before use.
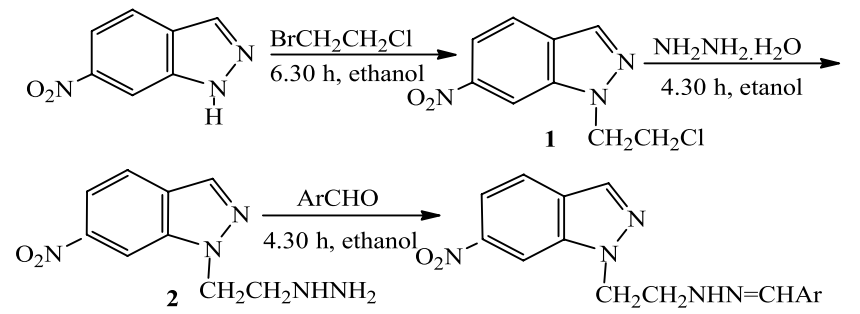

3(a-j)

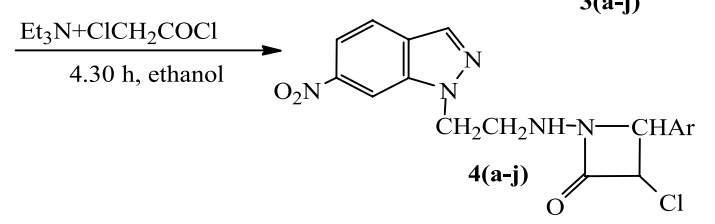

\begin{tabular}{cccc}
\multicolumn{4}{c}{ Ar $=$ substituted phenyl ring } \\
\hline Comp. & $\mathrm{Ar}$ & $\mathrm{Comp}$. & $\mathrm{Ar}$ \\
\hline $\mathbf{3 a}, \mathbf{4 a}$ & $\mathrm{C}_{6} \mathrm{H}_{5}$ & $\mathbf{3 f}, \mathbf{4 f}$ & $3-\mathrm{BrC}_{6} \mathrm{H}_{4}$ \\
$\mathbf{3 b}, \mathbf{4 b}$ & $4-\mathrm{ClC}_{6} \mathrm{H}_{4}$ & $\mathbf{3 g}, \mathbf{4 g}$ & $2-\mathrm{BrC}_{6} \mathrm{H}_{4}$ \\
$\mathbf{3 c}, \mathbf{4 c}$ & $3-\mathrm{ClC}_{6} \mathrm{H}_{4}$ & $\mathbf{3 h}, \mathbf{4 h}$ & $4-\mathrm{NO}_{2} \mathrm{C}_{6} \mathrm{H}_{4}$ \\
$\mathbf{3 d}, \mathbf{4 d}$ & $2-\mathrm{ClC}_{6} \mathrm{H}_{4}$ & $\mathbf{3 i}, \mathbf{4 i}$ & $3-\mathrm{NO}_{2} \mathrm{C}_{6} \mathrm{H}_{4}$ \\
$\mathbf{3 e}, \mathbf{4 e}$ & $4-\mathrm{BrC}_{6} \mathrm{H}_{4}$ & $\mathbf{3 j}, \mathbf{4 j}$ & $2-\mathrm{NO}_{2} \mathrm{C}_{6} \mathrm{H}_{4}$ \\
\hline
\end{tabular}

Scheme 1. Synthesis of compounds $1,2,3(a-j)$ and $4(a-j)$

\section{Procedure for the synthesis of compound 1}

6-Nitro- $1 \mathrm{H}$-indazole (0.308 mole) and 1-bromo-2-chloroethane $(0.308 \mathrm{~mole})$ in ethanol $(100 \mathrm{~mL})$ were stirred on a magnetic stirrer for about $6.30 \mathrm{~h}$ at room temperature. The completion of the reaction was 
monitored by silica gel-G coated TLC plates. After the completion of the reaction the product was filtered and purified over a silica gel packed column chromatography using $\mathrm{CHCl}_{3}: \mathrm{CH}_{3} \mathrm{OH}(8: 2 \mathrm{v} / \mathrm{v})$ system as eluant $(150 \mathrm{~mL})$. The purified product was dried under vacuo and recrystallized from acetone at room temperature to yield compound 1 (Figure 1).

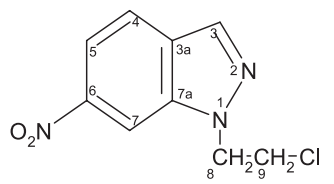

Figure 1. Structure of compound 1

\section{1-(2-chloroethyl)-6-nitro-1H-indazole 1}

Yield: $61 \%$, m.p. $154-155^{\circ} \mathrm{C}$; IR $\left(\mathrm{cm}^{-1}\right)$ : $768(\mathrm{C}-\mathrm{Cl}), 899(\mathrm{C}-\mathrm{N})$, $1326\left(\mathrm{~N}-\mathrm{CH}_{2}\right), 1532\left(\mathrm{NO}_{2}\right), 1572(\mathrm{C}=\mathrm{C}), 1448,2842,2889,\left(\mathrm{CH}_{2}\right)$, 3020 (CH-Ar); ${ }^{1} \mathrm{H}$ NMR $\left(\mathrm{CDCl}_{3}, 300 \mathrm{MHz}\right) \delta: 3.41$ (t, $2 \mathrm{H}, J=7.45$ $\mathrm{H}-9), 4.26$ (t, 2H, $J=7.45 \mathrm{~Hz}, \mathrm{H}-8), 7.86-8.35$ (m, 4H, Ar-H); ${ }^{13} \mathrm{C}$ NMR ( $\left.\mathrm{CDCl}_{3}, 75 \mathrm{MHz}\right) \delta$ : 42.8 (C-9), 47.1 (C-8), 118.7 (C-4), 120.4 (C-7), 122.2 (C-5), 126.1 (C-3a), 135.7 (C-7a), 136.2 (C-6), 137.0 (C-3); Anal. calcd for $\mathrm{C}_{9} \mathrm{H}_{8} \mathrm{~N}_{3} \mathrm{O}_{2} \mathrm{Cl}$ : C,47.90, $\mathrm{H}, 3.57, \mathrm{~N}, 18.62$; Found: $\mathrm{C}, 47.85, \mathrm{H}, 3.52, \mathrm{~N}, 18.56 \%$; Mass (FAB): 225M+.

\section{Procedure for the synthesis of compound 2}

Compound 1 ( 0.208 mole) and hydrazine hydrate $(0.208$ mole $)$ in ethanol $(100 \mathrm{~mL})$ were stirred on a magnetic stirrer for about $4.30 \mathrm{~h}$ at room temperature. The completion of the reaction was monitored by silica gel-G coated TLC plates. After the completion of the reaction the product was filtered and purified over a silica gel packed column chromatography using $\mathrm{CHCl}_{3}: \mathrm{CH}_{3} \mathrm{OH}(8: 2 \mathrm{v} / \mathrm{v})$ system as eluant (120 $\mathrm{mL})$. The purified product was dried under vacuo and recrystallized from acetone at room temperature to yield compound $\mathbf{2}$ (Figure 2).

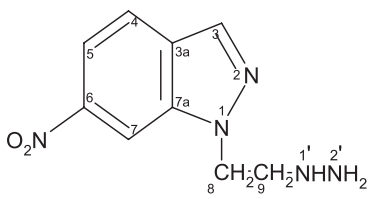

Figure 2. Structure of compound 2

\section{1-(2-hydrazinoethyl)-6-nitro-1H-indazole 2}

Yield: $72 \%$, m.p. $136-138^{\circ} \mathrm{C}$; IR $\left(\mathrm{cm}^{-1}\right)$ : $752(\mathrm{C}-\mathrm{Cl}), 872(\mathrm{C}-\mathrm{N})$, $1328\left(\mathrm{~N}-\mathrm{CH}_{2}\right), 1523\left(\mathrm{NO}_{2}\right), 1556(\mathrm{C}=\mathrm{C}), 1648(\mathrm{CO}), 1435,2839$, $2910\left(\mathrm{CH}_{2}\right), 3027(\mathrm{CH}-\mathrm{Ar}), 3342(\mathrm{NH}), 3456\left(\mathrm{NH}_{2}\right) ;{ }^{1} \mathrm{H} \mathrm{NMR}\left(\mathrm{CDCl}_{3}\right.$, $300 \mathrm{MHz}) \delta: 3.30-3.34$ (m, 2H, H-9), 4.18 (t, 2H, J = 7.45 Hz, H-8), 5.72 (s, 1H, H-1'), 5.92 (s, 2H, H-2'), 7.34-7.96 (m, 4H, Ar-H); ${ }^{13} \mathrm{C}$ NMR $\left(\mathrm{CDCl}_{3}, 75 \mathrm{MHz}\right)$ : 42.4 (C-9), 46.7 (C-8), 117.6 (C-4), 119.3 (C-7), 121.4 (C-5), 125.8 (C-3a), 134.5 (C-6), 136.7 (C-3), 139.2 (C-7a); Anal. calcd for $\mathrm{C}_{9} \mathrm{H}_{11} \mathrm{~N}_{5} \mathrm{O}_{2}$ : C,48.86, H,5.01, N,31.65; Found: C,48.82, H,5.00, N,31.61\%; Mass (FAB): 221M+.

\section{General procedure for the synthesis of compounds $3(a-j)$}

The compound 2 ( 0.026 mole) and benzaldehyde ( 0.026 mole $)$ in ethanol $(100 \mathrm{~mL})$ in the presence of 2-4 drops of glacial acetic acid were first stirred on a magnetic stirrer for about $2.00 \mathrm{~h}$ followed by reflux on a steam bath for about $2.30 \mathrm{~h}$. The completion of the reaction was monitored by silica gel-G coated TLC plates. The product was filtered and cooled at room temperature. The filtered product was purified over a silica gel packed column chromatography using $\mathrm{CH}_{3} \mathrm{OH}: \mathrm{CHCl}_{3}(7: 3 \mathrm{v} / \mathrm{v})$ as eluant $(80 \mathrm{~mL})$. The purified product was dried under vacuo and recrystallized from acetone at room temperature to furnish compound 3a (Figure 3).

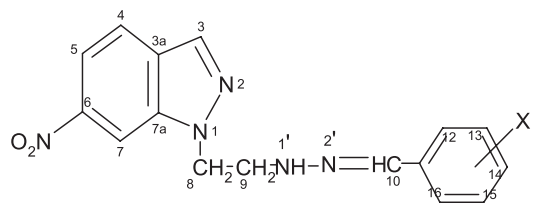

Figure 3. Structure of compound 3(a-j)

Compounds 3(b-j) have also been synthesized by using similar method as above.

\section{(E)-Benzaldehyde [2-(6-nitro-1H-indazol-1-yl)ethyl]hydrazone $3^{\text {a }}$}

Yield: $60 \%$, m.p. $155-156{ }^{\circ} \mathrm{C}$; IR $\left(\mathrm{cm}^{-1}\right): 742(\mathrm{C}-\mathrm{Cl}), 871(\mathrm{C}-\mathrm{N})$, $1328\left(\mathrm{~N}-\mathrm{CH}_{2}\right), 1523\left(\mathrm{NO}_{2}\right), 1534(\mathrm{C}=\mathrm{C}), 1555(\mathrm{~N}=\mathrm{CH}), 1650(\mathrm{C}=\mathrm{O})$, 1442, 2839, $2897\left(\mathrm{CH}_{2}\right), 3027(\mathrm{CH}-\mathrm{Ar}), 3356(\mathrm{NH}) ;{ }^{1} \mathrm{H} \mathrm{NMR}\left(\mathrm{CDCl}_{3}\right.$, $300 \mathrm{MHz}) \delta:$ 3.24-3.27 (m, 2H, H-9), 4.14 (t, 2H, J = $7.45 \mathrm{~Hz}, \mathrm{H}-8)$, 5.89 (s, 1H, H-1'), 7.98 (s, 1H, H-10), 7.22-7.97 (m, 9H, Ar-H); ${ }^{13} \mathrm{C}$ NMR $\left(\mathrm{CDCl}_{3}, 75 \mathrm{MHz}\right)$ 8: 38.4 (C-9), 45.3 (C-8), 115.7 (C-4), 119.4 (C-7), 120.5 (C-5), 121.5 (C-3a), 121.8 (C-12 and C-16), 122.4 (C-13 and C-15), 126.5 (C-14), 128.4 (C-11), 131.3 (C-6), 132.5 (C-3), 139.2 (C-7a), 150.6 (C-10); Anal. calcd for $\mathrm{C}_{16} \mathrm{H}_{15} \mathrm{~N}_{5} \mathrm{O}_{2}$ : C,62.12, $\mathrm{H}, 4.88$, $\mathrm{N}, 22.64$; Found: C,62.06, H,4.82, N,22.61\%; Mass (FAB): 309M+'.

\section{(E)-4-Chlorobenzaldehyde [2-(6-nitro-1H-indazol-1-yl)ethyl] hydrazone $3 \mathrm{~b}$}

Yield: 64\%, m.p. $160-162{ }^{\circ} \mathrm{C}$; IR $\left(\mathrm{cm}^{-1}\right): 745(\mathrm{C}-\mathrm{Cl}), 909(\mathrm{C}-\mathrm{N})$, $1347\left(\mathrm{~N}-\mathrm{CH}_{2}\right), 1537\left(\mathrm{NO}_{2}\right), 1574(\mathrm{C}=\mathrm{C}), 1583(\mathrm{~N}=\mathrm{CH}), 1638(\mathrm{C}=\mathrm{O})$, 1444, 2846, $2919\left(\mathrm{CH}_{2}\right), 3012$ (CH-Ar), $3346(\mathrm{NH}) ;{ }^{1} \mathrm{H}$ NMR $\left(\mathrm{CDCl}_{3}\right.$, $300 \mathrm{MHz}) \delta: 3.43-3.48$ (m, 2H, H-9), 4.24 (t, $2 \mathrm{H}, J=7.45 \mathrm{~Hz}, \mathrm{H}-8)$, 5.69 (s, 1H, H-1') 7.92 (s, 1H, H-10), 7.73-8.19 (m, 8H, Ar-H); ${ }^{13} \mathrm{C}$ NMR $\left(\mathrm{CDCl}_{3}, 75 \mathrm{MHz}\right)$ 8: 37.4 (C-9), 45.3 (C-8), 119.3 (C-4), 121.8 (C-7), 122.3 (C-5), 123.6 (C-3a), 124.9 (C-12 and C-16), 125.2 (C-13 and C-15), 128.3 (C-14), 129.8 (C-11), 131.3 (C-6), 135.7 (C-3), 140.5 (C-7a), 152.5 (C-10); Anal. calcd for $\mathrm{C}_{16} \mathrm{H}_{14} \mathrm{~N}_{5} \mathrm{O}_{2} \mathrm{Cl}$ : C,55.90, $\mathrm{H}, 4.10$, $\mathrm{N}, 20.37$; Found: C,55.87, H,4.03, N,20.31\%; Mass (FAB): $344 \mathrm{M}^{+}$.

\section{(E)-3-Chlorobenzaldehyde [2-(6-nitro-1H-indazol-1-yl)ethyl]} hydrazone $3 c$

Yield: 64\%, m.p. $158-160{ }^{\circ} \mathrm{C}$; IR $\left(\mathrm{cm}^{-1}\right): 753(\mathrm{C}-\mathrm{Cl}), 879(\mathrm{C}-\mathrm{N})$, $1338\left(\mathrm{~N}-\mathrm{CH}_{2}\right), 1534\left(\mathrm{NO}_{2}\right), 1545(\mathrm{C}=\mathrm{C}), 1553(\mathrm{~N}=\mathrm{CH}), 1655(\mathrm{C}=\mathrm{O})$, 1434, 2843, $2897\left(\mathrm{CH}_{2}\right), 3035(\mathrm{CH}-\mathrm{Ar}), 3364(\mathrm{NH}) ;{ }^{1} \mathrm{H} \mathrm{NMR}\left(\mathrm{CDCl}_{3}\right.$, $300 \mathrm{MHz}) \delta: 3.38-3.44$ (m, 2H, H-9), 4.28 (t, 2H, J=7.45 Hz, H-8), 5.67 (s, 1H, H-1'), 7.92 (s, 1H, H-10), 7.75-8.27 (m, 8H, Ar-H); ${ }^{13} \mathrm{C}$ $\mathrm{NMR}\left(\mathrm{CDCl}_{3}, 75 \mathrm{MHz}\right)$ 8: 42.4 (C-9), 45.4 (C-8), 117.4 (C-4), 121.2 (C-7), 121.7 (C-5), 122.7 (C-3a), 123.5 (C-12), 124.5 (C-16), 125.7 (C-13), 126.5 (C-15), 129.3 (C-14), 131.8 (C-11), 133.4 (C-6), 134.8 (C-3), 141.5 (C-7a), 154.6 (C-10); Anal. calcd for $\mathrm{C}_{16} \mathrm{H}_{14} \mathrm{~N}_{5} \mathrm{O}_{2} \mathrm{Cl}$ : C,55.90, H,4.10, N,20.37; Found: C,55.85, H,4.07, N,20.33\%; Mass (FAB): $344 \mathrm{M}^{+}$.

\section{(E)-2-Chlorobenzaldehyde [2-(6-nitro-1H-indazol-1-yl)ethyl] hydrazone 3d}

Yield: $65 \%$, m.p. $155-157^{\circ} \mathrm{C}$; IR $\left(\mathrm{cm}^{-1}\right): 746(\mathrm{C}-\mathrm{Cl}), 878(\mathrm{C}-\mathrm{N})$, $1344\left(\mathrm{~N}-\mathrm{CH}_{2}\right), 1529\left(\mathrm{NO}_{2}\right), 1537(\mathrm{C}=\mathrm{C}), 1566(\mathrm{~N}=\mathrm{CH}), 1661(\mathrm{C}=\mathrm{O})$, 1453, 2852, $2892\left(\mathrm{CH}_{2}\right), 3034(\mathrm{CH}-\mathrm{Ar}), 3360(\mathrm{NH}) ;{ }^{1} \mathrm{H} \mathrm{NMR}\left(\mathrm{CDCl}_{3}\right.$, 
$300 \mathrm{MHz}) \delta: 3.44-3.51(\mathrm{~m}, 2 \mathrm{H}, \mathrm{H}-9), 4.21(\mathrm{t}, 2 \mathrm{H}, J=7.40 \mathrm{~Hz}, \mathrm{H}-8)$, 5.64 (s, 1H, H-1'), 7.94 (s, 1H, H-10), 7.69-8.30 (m, 8H, Ar-H) ${ }^{13} \mathrm{C}$ NMR $\left(\mathrm{CDCl}_{3}, 75 \mathrm{MHz}\right) \delta$ : 42.6 (C-9), 48.3 (C-8), 117.6 (C-4), 119.0 (C-7), 122.2 (C-5), 123.6 (C-3a), 124.5 (C-12), 124.9 (C-16), 125.6 (C-13), 126.8 (C-15), 127.6 (C-14), 130.2 (C-11), 133.8 (C-6), 134.5 (C-3), 140.1 (C-7a), 152.7 (C-10); Anal. calcd for $\mathrm{C}_{16} \mathrm{H}_{14} \mathrm{~N}_{5} \mathrm{O}_{2} \mathrm{Cl}$ : C,55.90, H,4.10, N,20.37; Found: C,55.83, H,4.05, N,20.35\%; Mass(FAB): $344 \mathrm{M}^{+}$.

\section{(E)-4-Bromobenzaldehyde [2-(6-nitro-1H-indazol-1-yl)ethyl] hydrazone $3 \mathbf{e}$}

Yield: $66 \%$, m.p. $153-155^{\circ} \mathrm{C}$; IR $\left(\mathrm{cm}^{-1}\right): 884(\mathrm{C}-\mathrm{N}), 1342(\mathrm{~N}-$ $\left.\mathrm{CH}_{2}\right), 1527\left(\mathrm{NO}_{2}\right), 1534(\mathrm{C}=\mathrm{C}), 1568(\mathrm{~N}=\mathrm{CH}), 1654(\mathrm{C}=\mathrm{O}), 1459$, 2843, $2905\left(\mathrm{CH}_{2}\right), 3035(\mathrm{CH}-\mathrm{Ar}), 3369(\mathrm{NH}) ;{ }^{1} \mathrm{H} \mathrm{NMR}\left(\mathrm{CDCl}_{3}, 300\right.$ MHz) $\delta: 3.41-3.50$ (m, 2H, H-9), 4.33 (t, 2H, $J=7.50 \mathrm{~Hz}, \mathrm{H}-8), 5.65$ (s, 1H, H-1'), 7.93 (s, 1H, H-10), 7.74-8.26 (m, 8H, Ar-H); ${ }^{13} \mathrm{C}$ NMR $\left(\mathrm{CDCl}_{3}, 75 \mathrm{MHz}\right) \delta: 40.3$ (C-9), 47.5 (C-8), 116.7 (C-4), 119.4 (C-7), 120.8 (C-57), 123.5 (C-12 and C-16), 125.4 (C-3a), 126.4 (C-13 and C-15), 130.8 (C-14), 131.1 (C-11), 133.2 (C-6), 135.3 (C-3), 144.6 (C-7a), 154.3 (C-10); Anal. calcd for $\mathrm{C}_{16} \mathrm{H}_{14} \mathrm{~N}_{5} \mathrm{O}_{2} \mathrm{Br}$ : C,49.50, H,3.63, N18.03; Found: C,49.45, H,3.60, N,18.00\%; Mass (FAB): $388 \mathrm{M}^{+}$.

\section{(E)-3-Bromobenzaldehyde [2-(6-nitro-1H-indazol-1-yl)ethyl] hydrazone $3 f$}

Yield: 63\%, m.p. $149-151^{\circ} \mathrm{C}$; IR ( $\left.\mathrm{cm}^{-1}\right): 877(\mathrm{C}-\mathrm{N}), 1331\left(\mathrm{~N}-\mathrm{CH}_{2}\right)$, $1538\left(\mathrm{NO}_{2}\right), 1543(\mathrm{C}=\mathrm{C}), 1575(\mathrm{~N}=\mathrm{CH}), 1667(\mathrm{C}=\mathrm{O}), 1449,2843$, $2895\left(\mathrm{CH}_{2}\right), 3043$ (CH-Ar), $3368(\mathrm{NH}) ;{ }^{1} \mathrm{H} \mathrm{NMR}\left(\mathrm{CDCl}_{3}, 300 \mathrm{MHz}\right)$ $\delta: 3.46-3.52$ (m, 2H, H-9), 4.34 (t, 2H, $J=7.45 \mathrm{~Hz}, \mathrm{H}-8), 5.62(\mathrm{~s}, 1 \mathrm{H}$, H-1'), 7.89 (s, 1H, H-10), 7.64-8.36 (m, 8H, Ar-H); ${ }^{13} \mathrm{C} \mathrm{NMR}\left(\mathrm{CDCl}_{3}\right.$, $75 \mathrm{MHz})$ 8: 34.3 (C-9), 44.6 (C-8), 115.4 (C-4), 119.6 (C-7), 120.5 (C-5), 121.3 (C-3a), 122.8 (C-12), 124.5 (C-16), 125.3 (C-13), 126.3 (C-15), 128.6 (C-14), 131.7 (C-11), 132.8 (C-6), 133.9 (C-3), 143.2 (C-7a), 155.7 (C-10); Anal. Calcd for $\mathrm{C}_{16} \mathrm{H}_{14} \mathrm{~N}_{5} \mathrm{O}_{2} \mathrm{Br}: \mathrm{C}, 49.50, \mathrm{H}, 3.63$, N18.03; Found: C,49.44, H,3.53, N17.97\%; Mass (FAB): 488M+.

\section{(E)-2-Bromobenzaldehyde [2-(6-nitro-1H-indazol-1-yl)ethyl] hydrazone $3 \mathrm{~g}$}

Yield: $62 \%$, m.p. $146-148^{\circ} \mathrm{C}$; IR $\left(\mathrm{cm}^{-1}\right): 873(\mathrm{C}-\mathrm{N}), 1338\left(\mathrm{~N}-\mathrm{CH}_{2}\right)$, $1534\left(\mathrm{NO}_{2}\right), 1547(\mathrm{C}=\mathrm{C}), 1566(\mathrm{~N}=\mathrm{CH}), 1652(\mathrm{C}=\mathrm{O}), 1443,2856$, $2895\left(\mathrm{CH}_{2}\right), 3036(\mathrm{CH}-\mathrm{Ar}), 3372(\mathrm{NH}) ;{ }^{1} \mathrm{H} \mathrm{NMR}\left(\mathrm{CDCl}_{3}, 300 \mathrm{MHz}\right)$ $\delta: 3.47-3.52$ (m, 2H, H-9), 4.24 (t, 2H, $J=7.50 \mathrm{~Hz}, \mathrm{H}-8), 5.63(\mathrm{~s}, 1 \mathrm{H}$, H-1'), 7.95 (s, 1H, H-10), 7.80-8.22 (m, 8H, Ar-H); ${ }^{13} \mathrm{C} \mathrm{NMR}\left(\mathrm{CDCl}_{3}\right.$, $75 \mathrm{MHz})$ 8: 33.6 (C-9), 42.4 (C-8), 115.8 (C-4), 118.3 (C-7), 119.3 (C-5), 120.7 (C-3a), 122.6 (C-12), 123.3 (C-16), 125.2 (C-13), 126.7 (C-15), 130.5 (C-14), 132.7 (C-11), 135.3 (C-6), 137.8 (C-3), 141.4 (C-7a), 152.3 (C-10); Anal. calcd for $\mathrm{C}_{16} \mathrm{H}_{14} \mathrm{~N}_{5} \mathrm{O}_{2} \mathrm{Br}$ : C,49.50, H,3.63, N18.03; Found: C,49.42, H,3.56, N,17.93\%; Mass (FAB): $388 \mathrm{M}^{+}$.

\section{(E)-4-Nitrobenzaldehyde [2-(6-nitro-1H-indazol-1-yl)ethyl] hydrazone $3 \mathrm{~h}$}

Yield: 63\%, m.p. $168-170{ }^{\circ} \mathrm{C}$; IR $\left(\mathrm{cm}^{-1}\right): 753(\mathrm{C}-\mathrm{Cl}), 878(\mathrm{C}-\mathrm{N})$, $1340\left(\mathrm{~N}-\mathrm{CH}_{2}\right), 1528\left(\mathrm{NO}_{2}\right), 1537(\mathrm{C}=\mathrm{C}), 1562(\mathrm{~N}=\mathrm{CH}), 1665(\mathrm{C}=\mathrm{O})$, 1456, 2847, $2899\left(\mathrm{CH}_{2}\right), 3039$ (CH-Ar), $3359(\mathrm{NH}) ;{ }^{1} \mathrm{H} \mathrm{NMR}\left(\mathrm{CDCl}_{3}\right.$, $300 \mathrm{MHz}) \delta: 3.43-3.52$ (m, 2H, H-9), 4.32 (t, 2H, J = $7.40 \mathrm{~Hz}, \mathrm{H}-8)$, 5.56 (s, 1H, H-1'), 7.92 (s, 1H, H-10), 7.81-8.19 (m, 8H, Ar-H); ${ }^{13} \mathrm{C}$ NMR $\left(\mathrm{CDCl}_{3}, 75 \mathrm{MHz}\right) \delta$ : 43.1 (C-9), 45.4 (C-8), 113.6 (C-4), 120.9 (C-7), 121.2 (C-5), 122.5 (C-3a), 123.1 (C-12 and C-16), 124.4 (C-13 and C-15), 129.8 (C-14), 131.3 (C-11), 132.9 (C-6), 133.6 (C-3), 140.3 (C-7a), 155.7 (C-10); Anal. calcd for $\mathrm{C}_{16} \mathrm{H}_{14} \mathrm{~N}_{6} \mathrm{O}_{4}$ : C,54.23, H,3.98,
N,23.71; Found: C,54.20, H,3.95, N,23.67\%; Mass (FAB): 354M+.

(E)-3-Nitrobenzaldehyde [2-(6-nitro-1H-indazol-1-yl)ethyl] hydrazone 3i

Yield: 62\%, m.p. $164-166^{\circ} \mathrm{C}$; IR $\left(\mathrm{cm}^{-1}\right)$ : $747(\mathrm{C}-\mathrm{Cl}), 880(\mathrm{C}-$ $\mathrm{N}), 1335\left(\mathrm{~N}-\mathrm{CH}_{2}\right), 1533\left(\mathrm{NO}_{2}\right), 1539(\mathrm{C}=\mathrm{C}), 1562(\mathrm{~N}=\mathrm{CH}), 1656$ $(\mathrm{C}=\mathrm{O}), 1455,2848,2895\left(\mathrm{CH}_{2}\right), 3041(\mathrm{CH}-\mathrm{Ar}), 3356(\mathrm{NH}) ;{ }^{1} \mathrm{H} \mathrm{NMR}$ $\left(\mathrm{CDCl}_{3}, 300 \mathrm{MHz}\right) \delta: 3.48-3.52(\mathrm{~m}, 2 \mathrm{H}, \mathrm{H}-9), 4.34$ (t, 2H, $J=7.40$ Hz, H-8), 5.62 (s, 1H, H-1'), 7.94 (s, 1H, H-10), 7.70-8.22 (m, 8H, $\mathrm{Ar}-\mathrm{H}) ;{ }^{13} \mathrm{C} \mathrm{NMR}\left(\mathrm{CDCl}_{3}, 75 \mathrm{MHz}\right) \delta$ : 41.1 (C-9), 46.3 (C-8), 117.5 (C-4), 120.1 (C-7), 120.6 (C-5), 121.2 (C-3a), 122.4 (C-12), 123.5 (C-16), 124.3 (C-13), 125.8 (C-15), 126.3 (C-14), 127.4 (C-11), 131.5 (C-6), 134.2 (C-3), 139.1 (C-7a), 153.2 (C-10); Anal. calcd for $\mathrm{C}_{16} \mathrm{H}_{14} \mathrm{~N}_{6} \mathrm{O}_{4}$ : C,54.23, H,3.98, N,23.71; Found: C,54.20, H,3.94, $\mathrm{N}, 23.65 \%$; Mass (FAB): $354 \mathrm{M}^{+}$.

\section{(E)-2-Nitrobenzaldehyde [2-(6-nitro-1H-indazol-1-yl)ethyl] hydrazone $3 \mathbf{j}$}

Yield: 63\%, m.p. $165-167{ }^{\circ} \mathrm{C}$; IR $\left(\mathrm{cm}^{-1}\right)$ : $752(\mathrm{C}-\mathrm{Cl}), 877(\mathrm{C}-$ $\mathrm{N}), 1336\left(\mathrm{~N}-\mathrm{CH}_{2}\right), 1524\left(\mathrm{NO}_{2}\right), 1540(\mathrm{C}=\mathrm{C}), 1555(\mathrm{~N}=\mathrm{CH}), 1658$ $(\mathrm{C}=\mathrm{O}), 1446,2850,2899\left(\mathrm{CH}_{2}\right), 3034(\mathrm{CH}-\mathrm{Ar}), 3367(\mathrm{NH}) ;{ }^{1} \mathrm{H}$ NMR $\left(\mathrm{CDCl}_{3}, 300 \mathrm{MHz}\right) \delta: 3.40-3.46(\mathrm{~m}, 2 \mathrm{H}, \mathrm{H}-9), 4.21(\mathrm{t}, 2 \mathrm{H}, J=7.50$ Hz, H-8), 5.54 (s, 1H, H-1'), 7.89 (s, 1H, H-10), 7.73-8.26 (m, 8H, $\mathrm{Ar}-\mathrm{H}) ;{ }^{13} \mathrm{C} \mathrm{NMR}\left(\mathrm{CDCl}_{3}, 75 \mathrm{MHz}\right) \delta$ : 40.1 (C-9), 46.3 (C-8), 115.1 (C-4), 119.4 (C-7), 120.5 (C-5), 121.7 (C-12), 122.1 (C-3a), 122.8 (C-16), 123.2 (C-13), 123.7 (C-15), 126.6 (C-14), 127.2 (C-11), 130.4 (C-6), 131.7 (C-3), 138.7 (C-7a), 153.4 (C-10); Anal. calcd for $\mathrm{C}_{16} \mathrm{H}_{14} \mathrm{~N}_{6} \mathrm{O}_{4}$ : C,54.23, H,3.98, N,23.71; Found: C,54.19, H,3.92, $\mathrm{N}, 23.62 \%$; Mass (FAB): $354 \mathrm{M}^{+}$.

\section{General procedure for the synthesis of compounds 4(a-j)}

The compound 3a ( 0.008 mole) and chloroacetyl chloride $(0.008$ mole) were dissolved in ethanol $(100 \mathrm{~mL})$ in the presence of $\mathrm{Et}_{3} \mathrm{~N}$ ( 0.008 mole) were allowed to react at room temperature. The reaction mixture was first stirred on a magnetic stirrer for about $2.00 \mathrm{~h}$ followed by reflux on a steam bath for about $2.30 \mathrm{~h}$. The completion of the reaction was monitored by silica gel-G coated TLC plates. The product was filtered and cooled at room temperature. The filtered product was purified over a silica gel packed column chromatography using $\mathrm{CH}_{3} \mathrm{OH}: \mathrm{CHCl}_{3}(7: 3 \mathrm{v} / \mathrm{v})$ as eluant $(80 \mathrm{~mL})$. The purified product was dried under vacuo and recrystallized from acetone at room temperature to furnish compound $\mathbf{4 a}$ (Figure 4).

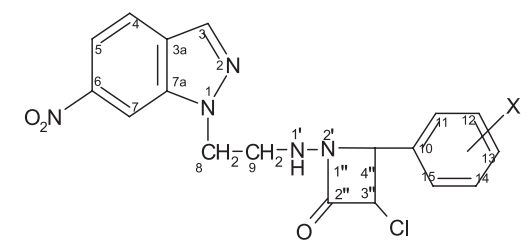

Figure 4. Structure of compound $\mathbf{4}(a-j)$

Compounds $\mathbf{4}(\mathbf{b}-\mathbf{j})$ have also been synthesized by using similar method as above.

\section{3-Chloro-1-\{[2-(6-nitro-1H-indazol-1-yl)ethyl]amino\}-4- phenyl-2-azetidinone $4 a$}

Yield: 66\%, m.p. $157-159^{\circ} \mathrm{C}$; IR $\left(\mathrm{cm}^{-1}\right)$ : $747(\mathrm{C}-\mathrm{Cl}), 875(\mathrm{C}-\mathrm{N})$, $1335\left(\mathrm{~N}-\mathrm{CH}_{2}\right), 1529\left(\mathrm{NO}_{2}\right), 1542(\mathrm{C}=\mathrm{C}), 1659(\mathrm{C}=\mathrm{O}), 1729(\mathrm{CO}$ cyclic), 1448, 2844, $2892\left(\mathrm{CH}_{2}\right), 2930(\mathrm{CH}-\mathrm{Cl}), 3025,(\mathrm{CH}-\mathrm{Ar}), 3360$ 
(NH); ${ }^{1} \mathrm{H}$ NMR $\left(\mathrm{CDCl}_{3}, 300 \mathrm{MHz}\right) \delta: 3.31-3.35$ (m, 2H, H-9), 4.24 (t, 2H, $J=7.45 \mathrm{~Hz}, \mathrm{H}-8$ ), 4.35 (d, 1H, $J=4.75 \mathrm{~Hz}, \mathrm{H}-3$ ”), 5.22 (d, $1 \mathrm{H}, J=4.75 \mathrm{~Hz}, \mathrm{H}-4$ ”'), 5.86 (s, 1H, H-1'), 7.10-7.74 (m, 9H, Ar-H); ${ }^{13} \mathrm{C} \mathrm{NMR}\left(\mathrm{CDCl}_{3}, 75 \mathrm{MHz}\right) \delta: 38.9$ (C-9), 46.2 (C-8), 50.1 (C-3"), 61.4 (C-4”), 116.2 (C-4), 121.7 (C-7), 122.3 (C-5), 126.6 (C-11 and C-15), 127.1 (C-3a), 128.2 (C-13), 129.1 (C-12 and C-14), 132.5 (C-6), 134.8 (C-3), 135.2 (C-10), 140.4 (C-7a), 168.1 (C-2”); Anal. calcd for $\mathrm{C}_{18} \mathrm{H}_{16} \mathrm{~N}_{5} \mathrm{O}_{3} \mathrm{Cl}$ : C,56.03, H,4.18, N,18.15; Found: C,56.00, $\mathrm{H}, 4.12, \mathrm{~N}, 18.10 \%$; Mass (FAB): $386 \mathrm{M}^{+}$.

\section{3-Chloro-1-\{[2-(6-nitro-1H-indazol-1-yl)ethyl]amino\}-4-(4- chlorophenyl)-2-azetidinone $4 \mathrm{~b}$}

Yield: $65 \%$, m.p. $161-162{ }^{\circ} \mathrm{C}$; IR $\left(\mathrm{cm}^{-1}\right)$ : $759(\mathrm{C}-\mathrm{Cl}), 885(\mathrm{C}-\mathrm{N})$, $1337\left(\mathrm{~N}-\mathrm{CH}_{2}\right), 1531\left(\mathrm{NO}_{2}\right), 1545(\mathrm{C}=\mathrm{C}), 1662(\mathrm{C}=\mathrm{O}), 1735(\mathrm{CO}$ cyclic), 1450, 2849, $2895\left(\mathrm{CH}_{2}\right), 2932$ (CH-Cl), 3032, (CH-Ar), 3365 (NH); ${ }^{1} \mathrm{H}$ NMR $\left(\mathrm{CDCl}_{3}, 300 \mathrm{MHz}\right) \delta: 3.31-3.36$ (m, 2H, H-9), 4.22 (t, $2 \mathrm{H}, J=7.35 \mathrm{~Hz}, \mathrm{H}-8$ ), 4.62 (d, $1 \mathrm{H}, J=4.80 \mathrm{~Hz}, \mathrm{H}-3$ "), 5.36 (d, $1 \mathrm{H}, J=4.80 \mathrm{~Hz}, \mathrm{H}-4$ "), 5.77 (s, 1H, H-1'), 7.16-7.82 (m, 8H, Ar-H); ${ }^{13} \mathrm{C} \mathrm{NMR}\left(\mathrm{CDCl}_{3}, 75 \mathrm{MHz}\right) \delta: 39.6$ (C-9), 49.4 (C-8), 52.7 (C-3"), 65.4 (C-4"), 120.1 (C-4), 122.6 (C-7), 123.2 (C-5), 128.6 (C-11 and C-15), 129.4 (C-3a), 130.7 (C-13), 132.5 (C-12 and C-14), 135.0 (C-6), 135.6 (C-10), 136.7 (C-3), 142.6 (C-7a), 170.5 (C-2”); Anal. calcd for $\mathrm{C}_{18} \mathrm{H}_{15} \mathrm{~N}_{5} \mathrm{O}_{3} \mathrm{Cl}_{2}$ : C,51.44, $\mathrm{H}, 3.59$, N, 16.66; Found: C,51.42, $\mathrm{H}, 3.54, \mathrm{~N}, 16.61 \%$; Mass(FAB): 420M+'.

3-Chloro-1-\{[2-(6-nitro-1H-indazol-1-yl)ethyl]amino $\}-4-(3-$ chlorophenyl)-2-azetidinone $4 \mathrm{c}$

Yield: $62 \%$, m.p. $160-162^{\circ} \mathrm{C}$; IR $\left(\mathrm{cm}^{-1}\right)$ : $755(\mathrm{C}-\mathrm{Cl}), 876(\mathrm{C}-\mathrm{N})$, $1340\left(\mathrm{~N}-\mathrm{CH}_{2}\right), 1533\left(\mathrm{NO}_{2}\right), 1547(\mathrm{C}=\mathrm{C}), 1665(\mathrm{C}=\mathrm{O}), 1740(\mathrm{CO}$ cyclic), 1454, 2852, 2901 ( $\left.\mathrm{CH}_{2}\right), 2935$ (CH-Cl), 3028, (CH-Ar), 3362 $(\mathrm{NH}) ;{ }^{1} \mathrm{H} \mathrm{NMR}\left(\mathrm{CDCl}_{3}, 300 \mathrm{MHz}\right) \delta: 3.31-3.38$ (m, 2H, H-9), 4.20 (t, $2 \mathrm{H}, J=7.35 \mathrm{~Hz}, \mathrm{H}-8), 4.68$ (d, 1H, $J=5.00 \mathrm{~Hz}, \mathrm{H}-3$ ”), 5.36 (d, 1H, $J=5.00 \mathrm{~Hz}, \mathrm{H}-4$ "), 5.74 (s, 1H, H-1'), 6.79-7.94 (m, 8H, Ar-H); ${ }^{13} \mathrm{C}$ NMR $\left(\mathrm{CDCl}_{3}, 75 \mathrm{MHz}\right) \delta: 41.7$ (C-9), 50.9 (C-8), 52.6 (C-3”), 65.7 (C-4”), 116.9 (C-4), 121.1 (C-7), 122.5 (C-5), 129.5 (C-11), 129.8 (C-15), 130.4 (C-3a), 131.6 (C-13), 132.2 (C-12), 133.7 (C-14), 136.9 (C-6), 137.1 (C-3), 138.2 (C-10), 141.7 (C-7a), 169.7 (C-2”); Anal. calcd for $\mathrm{C}_{18} \mathrm{H}_{15} \mathrm{~N}_{5} \mathrm{O}_{3} \mathrm{Cl}_{2}$ : C,51.44, H,3.59, N,16.66; Found: C,51.40, $\mathrm{H}, 3.55, \mathrm{~N}, 16.61 \%$; Mass (FAB): 420M+'.

\section{3-Chloro-1-\{[2-(6-nitro-1H-indazol-1-yl)ethyl]amino $\}$-4-(2- chlorophenyl)-2-azetidinone 4d}

Yield: $66 \%$, m.p. $161-163{ }^{\circ} \mathrm{C}$; IR $\left(\mathrm{cm}^{-1}\right): 751(\mathrm{C}-\mathrm{Cl}), 880(\mathrm{C}-\mathrm{N})$, $1348\left(\mathrm{~N}-\mathrm{CH}_{2}\right), 1534\left(\mathrm{NO}_{2}\right), 1546(\mathrm{C}=\mathrm{C}), 1665(\mathrm{C}=\mathrm{O}), 1738(\mathrm{CO}$ cyclic), 1448, 2853, $2903\left(\mathrm{CH}_{2}\right), 2940$ (CH-Cl), 3027 (CH-Ar), 3370 $(\mathrm{NH}) ;{ }^{1} \mathrm{H} \mathrm{NMR}\left(\mathrm{CDCl}_{3}, 300 \mathrm{MHz}\right) \delta: 3.30-3.36(\mathrm{~m}, 2 \mathrm{H}, \mathrm{H}-9), 4.23(\mathrm{t}$, $2 \mathrm{H}, J=7.35 \mathrm{~Hz}, \mathrm{H}-8), 4.53$ (d, 1H, $J=4.90 \mathrm{~Hz}, \mathrm{H}-3$ ”), 5.30 (d, 1H, $J=4.90 \mathrm{~Hz}, \mathrm{H}-4$ "), 5.72 (s, 1H, H-1'), 6.81-7.82 (m, 8H, Ar-H); ${ }^{13} \mathrm{C}$ NMR $\left(\mathrm{CDCl}_{3}, 75 \mathrm{MHz}\right) \delta$ : 40.6 (C-9), 48.8 (C-8), 53.5 (C-3”), 62.6 (C-4"), 119.7 (C-4), 123.5 (C-7), 124.3 (C-5), 126.9 (C-11), 127.0 (C-15), 127.4 (C-3a), 128.6 (C-13), 129.7 (C-12), 130.2 (C-14), 133.5 (C-6), 134.3 (C-3), 137.5 (C-10), 142.3 (C-7a), 171.8 (C-2”); Anal. calcd for $\mathrm{C}_{18} \mathrm{H}_{15} \mathrm{~N}_{5} \mathrm{O}_{3} \mathrm{Cl}_{2}$ : C,51.44, $\mathrm{H}, 3.59, \mathrm{~N}, 16.66$; Found: C,51.42, $\mathrm{H}, 3.55, \mathrm{~N}, 16.61 \%$; Mass (FAB): $420 \mathrm{M}^{+}$.

\section{3-Chloro-1-\{[2-(6-nitro-1H-indazol-1-yl)ethyl]amino\}-4-(4- bromophenyl)-2-azetidinone $4 \mathrm{e}$}

Yield: 62\%, m.p. 159-161 C; IR (cm $\left.{ }^{-1}\right)$ : $578(\mathrm{C}-\mathrm{Br}), 886(\mathrm{C}-\mathrm{N})$, $1342\left(\mathrm{~N}-\mathrm{CH}_{2}\right), 1540\left(\mathrm{NO}_{2}\right), 1548(\mathrm{C}=\mathrm{C}), 1662(\mathrm{C}=\mathrm{O}), 1736(\mathrm{CO}$ cyclic), 1456, 2854, $2897\left(\mathrm{CH}_{2}\right), 2942(\mathrm{CH}-\mathrm{Cl}), 3032,(\mathrm{CH}-\mathrm{Ar}), 3368$ (NH); ${ }^{1} \mathrm{H}$ NMR $\left(\mathrm{CDCl}_{3}, 300 \mathrm{MHz}\right) \delta: 3.39-3.45$ (m, 2H, H-9), 4.28 (t, 2H, $J=7.35 \mathrm{~Hz}, \mathrm{H}-8), 4.62$ (d, 1H, $J=5.00 \mathrm{~Hz}, \mathrm{H}-3$ ”), 5.32 (d, $1 \mathrm{H}, J=5.00 \mathrm{~Hz}, \mathrm{H}-4$ "), 5.79 (s, 1H, H-1'), 7.25-7.95 (m, 8H, Ar-H); ${ }^{13} \mathrm{C} \mathrm{NMR}\left(\mathrm{CDCl}_{3}, 75 \mathrm{MHz}\right) \delta$ : 42.9 (C-9), 47.6 (C-8), 51.5 (C-3”), 64.4 (C-4”), 117.3 (C-4), 120.7 (C-7), 121.6 (C-5), 128.7 (C-11 and C-15), 129.6 (C-3a), 130.5 (C-13), 131.7 (C-12 and C-14), 134.3 (C-6), 135.7 (C-3), 138.5 (C-10), 141.6 (C-7a), 172.5 (C-2”); Anal. calcd for $\mathrm{C}_{18} \mathrm{H}_{15} \mathrm{~N}_{5} \mathrm{O}_{3} \mathrm{BrCl}$ : C,46.52, $\mathrm{H}, 3.25, \mathrm{~N}, 15.07$; Found: $\mathrm{C}, 46.45$, H,3.20, N, 15.01\%; Mass (FAB): 465 $\mathrm{M}^{+}$.

\section{3-Chloro-1-\{[2-(6-nitro-1H-indazol-1-yl)ethyl]amino\}-4-(3- bromophenyl)-2-azetidinone $4 \mathrm{f}$}

Yield: $63 \%$, m.p. $155-157^{\circ} \mathrm{C}$; IR ( $\left.\mathrm{cm}^{-1}\right)$ : $571(\mathrm{C}-\mathrm{Br}), 877(\mathrm{C}-\mathrm{N})$, $1343\left(\mathrm{~N}-\mathrm{CH}_{2}\right), 1536\left(\mathrm{NO}_{2}\right), 1550(\mathrm{C}=\mathrm{C}), 1663(\mathrm{C}=\mathrm{O}), 1737(\mathrm{CO}$ cyclic), 1456, 2851, $2896\left(\mathrm{CH}_{2}\right), 2943$ (CH-Cl), 3035, (CH-Ar), 3364 $(\mathrm{NH}) ;{ }^{1} \mathrm{H} \mathrm{NMR}\left(\mathrm{CDCl}_{3}, 300 \mathrm{MHz}\right) \delta: 3.34-3.38$ (m, 2H, H-9), $4.26(\mathrm{t}$, $2 \mathrm{H}, J=7.35 \mathrm{~Hz}, \mathrm{H}-8), 4.45$ (d, 1H, $J=4.90 \mathrm{~Hz}, \mathrm{H}-3$ ”), 5.37 (d, 1H, $J=4.90 \mathrm{~Hz}, \mathrm{H}-4$ "), 5.77 (s, 1H, H-1'), 7.21-7.92 (m, 8H, Ar-H); ${ }^{13} \mathrm{C}$ NMR ( $\left.\mathrm{CDCl}_{3}, 75 \mathrm{MHz}\right) \delta: 39.8$ (C-9), 49.3 (C-8), 51.4 (C-3”), 63.3 (C-4”), 119.6 (C-4), 122.6 (C-7), 123.8 (C-5), 129.7 (C-11), 130.1 (C-15), 130.8 (C-3a), 131.5 (C-13), 132.3 (C-12), 133.7 (C-14), 135.8 (C-6), 136.6 (C-10), 137.5 (C-3), 142.3 (C-7a), 172.7 (C-2”); Anal. calcd for $\mathrm{C}_{18} \mathrm{H}_{15} \mathrm{~N}_{5} \mathrm{O}_{3} \mathrm{BrCl}$ : C, 46.52, $\mathrm{H}, 3.25, \mathrm{~N}, 15.07$; Found: C,46.47, H,3.21, N,15.22\%; Mass (FAB): 465M+.

\section{3-Chloro-1-\{[2-(6-nitro-1H-indazol-1-yl)ethyl]amino\}-4-(2- bromophenyl)-2-azetidinone $4 \mathrm{~g}$}

Yield: $62 \%$, m.p. $156-158^{\circ} \mathrm{C}$; IR $\left(\mathrm{cm}^{-1}\right)$ : $565(\mathrm{C}-\mathrm{Br}), 758(\mathrm{C}-\mathrm{Cl})$, $883(\mathrm{C}-\mathrm{N}), 1346\left(\mathrm{~N}-\mathrm{CH}_{2}\right), 1535\left(\mathrm{NO}_{2}\right), 1546(\mathrm{C}=\mathrm{C}), 1664(\mathrm{C}=\mathrm{O})$, 1738 (CO cyclic), 1452, 2846, $2900\left(\mathrm{CH}_{2}\right), 2941(\mathrm{CH}-\mathrm{Cl}), 3028$, (CH-Ar), $3361(\mathrm{NH}) ;{ }^{1} \mathrm{H}$ NMR $\left(\mathrm{CDCl}_{3}, 300 \mathrm{MHz}\right) \delta: 3.31-3.39$ (m, $2 \mathrm{H}, \mathrm{H}-9), 4.25$ (t, 2H, $J=7.35 \mathrm{~Hz}, \mathrm{H}-8), 4.44$ (d, $1 \mathrm{H}, J=5.10 \mathrm{~Hz}$, H-3”), 5.35 (d, 1H, J = 5.10 Hz, H-4”), 5.72 (s, 1H, H-1'), 7.27-7.84 (m, 8H, Ar-H); ${ }^{13} \mathrm{C} \mathrm{NMR}\left(\mathrm{CDCl}_{3}, 75 \mathrm{MHz}\right) \delta: 40.2$ (C-9), 50.5 (C-8), 53.2 (C-3”), 61.8 (C-4”), 118.4 (C-4), 124.7 (C-7), 125.4 (C-5), 127.6 (C-11), 128.2 (C-15), 128.5 (C-3a), 129.2 (C-13), 130.6 (C-12), 131.2 (C-14), 133.7 (C-6), 134.6 (C-3), 138.1 (C-10), 139.2 (C-7a), 169.3 (C-2"); Anal. calcd for $\mathrm{C}_{18} \mathrm{H}_{15} \mathrm{~N}_{5} \mathrm{O}_{3} \mathrm{BrCl}$ : C,46.52, H,3.25, N, 15.07; Found: C,46.42, H,3.20, N,15.01\%; Mass (FAB): 465M+.

\section{3-Chloro-1-\{[2-(6-nitro-1H-indazol-1-yl)ethyl]amino\}-4-(4- nitrophenyl)-2-azetidinone $4 \mathrm{~h}$}

Yield: $65 \%$, m.p. $169-171^{\circ} \mathrm{C}$; IR $\left(\mathrm{cm}^{-1}\right): 869$ (C-NO), $756(\mathrm{C}-\mathrm{Cl})$, $879(\mathrm{C}-\mathrm{N}), 1349\left(\mathrm{~N}-\mathrm{CH}_{2}\right), 1541\left(\mathrm{NO}_{2}\right), 1546(\mathrm{C}=\mathrm{C}), 1666(\mathrm{C}=\mathrm{O})$, 1740 (CO cyclic), 1454, 2845, $2904\left(\mathrm{CH}_{2}\right), 2940$ (CH-Cl), 3024, (CH-Ar), $3363(\mathrm{NH}) ;{ }^{1} \mathrm{H}$ NMR $\left(\mathrm{CDCl}_{3}, 300 \mathrm{MHz}\right)$ 8: 3.34-3.38 (m, 2H, H-9), 4.29 (t, 2H, $J=7.35 \mathrm{~Hz}, \mathrm{H}-8), 4.38$ (d, $1 \mathrm{H}, J=4.80 \mathrm{~Hz}$, H-3”), 5.27 (d, 1H, J = 4.80 Hz, H-4”), 5.75 (s, 1H, H-1'), 7.10-7.71 (m, 8H, Ar-H); ${ }^{13} \mathrm{C} \mathrm{NMR}\left(\mathrm{CDCl}_{3}, 75 \mathrm{MHz}\right) \delta: 42.1$ (C-9), $46.6(\mathrm{C}-8)$, 52.3 (C-3”), 64.4 (C-4”), 120.7 (C-4), 123.5 (C-7), 124.3 (C-5), 126.7 (C-11 and C-15), 127.8 (C-3a), 128.2 (C-13), 129.4 (C-12 and C-14), 132.6 (C-6), 134.6 (C-3), 139.4 (C-10), 141.2 (C-7a), 170.7 (C-2”); Anal. calcd for $\mathrm{C}_{18} \mathrm{H}_{15} \mathrm{~N}_{6} \mathrm{O}_{5} \mathrm{Cl}$ : C,50.18, H,3.50, N,19.50; Found: C,50.12, H,3.45, N,19.52\%; Mass (FAB): 431 M+.

\section{3-Chloro-1-\{[2-(6-nitro-1H-indazol-1-yl)ethyl]amino\}-4-(3- nitrophenyl)-2-azetidinone $4 \mathrm{i}$}

Yield: 64\%, m.p. $167-169^{\circ} \mathrm{C}$; IR ( $\left.\mathrm{cm}^{-1}\right)$ : 750 (C-Cl), 862 (C-NO), 
$1350\left(\mathrm{~N}-\mathrm{CH}_{2}\right), 1538\left(\mathrm{NO}_{2}\right), 1545(\mathrm{C}=\mathrm{C}), 1665(\mathrm{C}=\mathrm{O}), 1739(\mathrm{CO}$ cyclic), 1455, 2849, $2899\left(\mathrm{CH}_{2}\right), 2946$ (CH-Cl), 3027, (CH-Ar), 3366 $(\mathrm{NH}) ;{ }^{1} \mathrm{H}$ NMR $\left(\mathrm{CDCl}_{3}, 300 \mathrm{MHz}\right) \delta: 3.30-3.35$ (m, 2H, H-9), $4.19(\mathrm{t}$, $2 \mathrm{H}, J=7.35 \mathrm{~Hz}, \mathrm{H}-8), 4.24$ (d, 1H, $J=4.80 \mathrm{~Hz}, \mathrm{H}-3$ "), 5.32 (d, $1 \mathrm{H}$, $J=4.80 \mathrm{~Hz}, \mathrm{H}-4$ ") 5.78 (s, 1H, H-1'), 6.90-7.74 (m, 8H, Ar-H); ${ }^{13} \mathrm{C}$ NMR (CDCl, $75 \mathrm{MHz})$ 8: 40.4 (C-9), 47.8 (C-8), 50.2 (C-3”), 63.3 (C-4”), 117.7 (C-4), 122.5 (C-5), 123.6 (C-7), 129.6 (C-11), 130.1 (C-15), 130.6 (C-3a), 131.5 (C-13), 132.7 (C-12), 132.9 (C-14), 135.5 (C-6), 136.1 (C-3), 137.9 (C-10), 140.6 (C-7a), 171.2 (C-2”); Anal. calcd for $\mathrm{C}_{18} \mathrm{H}_{15} \mathrm{~N}_{6} \mathrm{O}_{5} \mathrm{Cl}$ : C,50.18, H,3.50, N,19.50; Found: C,50.15, $\mathrm{H}, 3.40, \mathrm{~N}, 19.46 \%$; Mass (FAB): 431M+.

\section{3-Chloro-1-\{[2-(6-nitro-1H-indazol-1-yl)ethyl]amino\}-4-(2- nitrophenyl)-2-azetidinone $4 \mathrm{j}$}

Yield: $62 \%$, m.p. $164-165^{\circ} \mathrm{C}$; IR $\left(\mathrm{cm}^{-1}\right)$ : $757(\mathrm{C}-\mathrm{Cl}), 869$ (C-NO), $1347\left(\mathrm{~N}-\mathrm{CH}_{2}\right), 1534\left(\mathrm{NO}_{2}\right), 1547(\mathrm{C}=\mathrm{C}), 1663(\mathrm{C}=\mathrm{O}), 1741(\mathrm{CO}$ cyclic), 1460, 2850, $2896\left(\mathrm{CH}_{2}\right), 2945$ (CH-Cl), 3030, (CH-Ar), 3367 (NH); ${ }^{1} \mathrm{H} \mathrm{NMR}\left(\mathrm{CDCl}_{3}, 300 \mathrm{MHz}\right) \delta: 3.34-3.39$ (m, 2H, H-9), $4.29(\mathrm{t}$, $2 \mathrm{H}, J=7.35 \mathrm{~Hz}, \mathrm{H}-8$ ), 4.41 (d, 1H, $J=4.80 \mathrm{~Hz}, \mathrm{H}-3$ ”), 5.34 (d, 1H, $J=4.80 \mathrm{~Hz}, \mathrm{H}-4$ "), 5.74 (s, 1H, H-1'), 7.05-7.71 (m, 8H, Ar-H); ${ }^{13} \mathrm{C}$ NMR (CDCl, $75 \mathrm{MHz})$ \&: 41.6 (C-9), 48.7 (C-8), 50.3 (C-3"), 62.6 (C-4”), 118.8 (C-4), 124.5 (C-7), 125.8 (C-5), 127.6 (C-11), 127.9 (C-15), 128.3 (C-3a), 129.2 (C-13), 130.7 (C-12), 132.1 (C-14), 134.1 (C-6), 135.5 (C-3), 139.4 (C-10), 140.4 (C-7a), 168.9 (C-2”); Anal. calcd for $\mathrm{C}_{18} \mathrm{H}_{15} \mathrm{~N}_{6} \mathrm{O}_{5} \mathrm{Cl}$ : C,50.18, H,3.50, N,19.50; Found: C,50.11, $\mathrm{H}, 3.42, \mathrm{~N}, 19.44 \%$; Mass (FAB): $431 \mathrm{M}^{+}$.

\section{Biological study}

Antibacterial, antifungal and antitubercular activities

The antibacterial, antifungal and antitubercular activities of compounds $\mathbf{4}(\mathbf{a}-\mathbf{j})$ have been assayed in vitro against selected bacteria, Bacillus subtilis, Escherichia coli, Staphylococcus aureus, Klebsiella pneumoniae, fungi, Aspergillus niger, Aspergillus flavus, Candida albicans Fusarium oxisporium and Mycobacterium tuberculosis H37Rv strain respectively. MIC value of compounds $4(\mathbf{a}-\mathbf{j})$ were determined using filter paper disc diffusion method (antibacterial and antifungal activities) and L. J. medium (Conventional) method (antitubercular activity). Streptomycin and Griseofulvin used as standard for antibacterial and antifungal activities showed MIC range for all bacterial strain 1.25-3.25 $\mu \mathrm{g} / \mathrm{mL}$ and for all fungal strain $6.25-12.5$ $\mu \mathrm{g} / \mathrm{mL}$ respectively and for antitubercular activity, Isoniazid and Rifampicin taken as standards (MIC range 1.25-2.50 $\mu \mathrm{g} / \mathrm{mL}$ ). All standards also screened under the similar condition for comparison. All concentrations used in $\mu \mathrm{g} / \mathrm{mL}$. Results of all given activities of above compounds were given in Table 1 .

\section{Antiinflammatory activity}

Carageenan induced rat paw oedema method was employed for evaluating the antiinflammatory activity of compounds at a dose 50 $\mathrm{mg} / \mathrm{kg}$ bw in albino rats (weighing 80-110 gm, each group contain 5 animal) using phenylbutazone as a standard drug for comparison at a dose $30 \mathrm{mg} / \mathrm{kg}$ bw. The rate paw oedema was produced by the method of Winter et al.. ${ }^{15}$ The percentage inhibition of inflammation was calculated by applying Newbould formula. Results of the compounds $\mathbf{4}(\mathbf{a}-\mathbf{j})$ were given in Table 2 .

\section{ACKNOWLEDGEMENT}

The authors are thankful to SAIF, Central Drugs Research Institute, Lucknow (India) for providing spectral and analytical data of the compounds. We are thankful to Head, Department of Biotechnology, Dr. H. S. Gour, University, Sagar (India) for antimicrobial (antibacterial and antifungal) and Microcare Laboratory and Tuberculosis Research Center, Surat, Gujrat (India) for antituberculosis activity. We are also thankful to Head, Department of Chemistry, Dr. H. S. Gour, University, Sagar (India) for giving the facilities to carryout the work.

\section{REFERENCES}

1. Gerpe, A.; Aguirre, G.; Boiani, L.; Cerecetto, H.; Gonzalez, M.; OleaAzar, C.; Rigol, C.; Maya, J. D.; Morello, A.; Piro, O. E.; Aran, V. J.; Azqueta, A.; Cerain, A. L.; Monge, A.; Rojas, M. A.; Yaluff, G.; Bioorg. Med. Chem. 2006, 14, 3467.

2. Berhe, S.; Slupe, A.; Luster, C.; Charlier, H. A.; Warner, D. L.; Zalkow, L. H.; Burgess, E. M.; Enwerem, N. M.; Bakare, O.; Bioorg. Med. Chem. 2010, 18, 134.

3. Tanaka, H.; Tanizawa, K.; Arai, T.; Saito, K.; Soda, K.; FEBS Lett. 1986, 196, 357.

4. Rosati, O.; Curini, M.; Marcotullio, M. C.; Macchiarulo, A.; Perfumi, M.; Mattioli, L.; Rismondo, F.; Cravotto, G.; Bioorg. Med. Chem. 2007, $15,3463$.

5. Bovens, S.; Kaptur, M.; Elfringhoff, A. S.; Lehr, M.; Bioorg. Med. Chem. Lett. 2009, 19, 2107.

6. Cottyn, B.; Acher, F.; Ramassamy, B.; Alvey, L.; Lepoivre, M.; Frapart, Y.; Stuehr, D.; Mansuy, D.; Boucher, J.-L.; Vichard, D.; Bioorg. Med. Chem. 2008, 16, 5962.

7. Raffa, D.; Maggio, B.; Cascioferro, S.; Raimondi, M. V.; Schillaci, D.; Gallo, G.; Daidone, G.; Plescia, S.; Meneghetti, F.; Bombieri, G.; Cristina, A. D.; Pipitone, R. M.; Grimaudo, S.; Tolomeo, M.; Eur. J. Med. Chem. 2009, 44, 165.

8. Aki, C.; Chao, J.; Ferreira, J. A.; Dwyer, M. P.; Yu, Y.; Chao, J.; Merritt, R. J.; Lai; G.; Wu, M.; Hipkin, R. W.; Fan, X.; Gonsiorek, W.; Fosseta, J.; Rindgen, D.; Fine, J.; Lundell, D.; Taveras, A. G.; Biju, P.; Bioorg. Med. Chem. Lett. 2009, 19, 4446.

9. Halve, A. K.; Bhadauria, D.; Dubey, R.; Bioorg. Med. Chem. Lett. 2007, $17,341$.

10. Upadhyay, A.; Srivastava, S. K.; Srivastava, S. D.; Yadav, R.; Proc. Natl. Acad. Sci. India 2010, 80, 131.

11. Upadhyay, A.; Srivastava, S. K.; Srivastava, S. D.; Yadav, R.; Indian J. Chem. 2011, 50B, 89.

12. Vasoya, S. L.; Patel, M. R.; Dobaria, S. V.; Joshi,, H. S.; Indian J. Chem. 2005, 44B, 405

13. Gerona-Navarro, G.; Vega, M. J. P. D.; Garcia-Lopez, M. T.; Andrei, G.; Snoeck, R.; Balzaraini, J.; Clercq, E. D.; Gonzalez-Muniz, R.; Bioorg. Med. Chem. Lett. 2004, 14, 2253.

14. Upadhyay, A.; Srivastava, S. K.; Srivastava, S. D.; Synth. Commun.2011, 41, 2544.

15. Winter, C. A.; Risley, E. A.; Nuss, G. W.; Proc. Soc. Exp. Biol. Med. 1962, 3, 544 . 